\title{
Mesalamine-Induced Myopericarditis: A Case Report and Literature Review
}

\author{
Mohamed E. Taha ${ }^{\mathrm{a}, \mathrm{b}}$, Abubaker Abdalla ${ }^{\mathrm{a}}$, Jaafar Al-Khafaji ${ }^{\mathrm{a}}$, Samira Malik
}

\begin{abstract}
Inflammation of the myocardium (myocarditis) or pericardium (pericarditis) or both (myopericarditis) as side effects of mesalamine, a drug widely used in the treatment of inflammatory bowel disease, is a rare, but potentially lethal complication. We report a case of myopericarditis occurring in a young Caucasian woman 14 days following initiation of mesalamine therapy for treatment of a newly diagnosed ulcerative colitis (UC). She presented with pleuritic chest pain, elevated troponin levels and pre-syncope. The diagnosis of myopericarditis was made based on the clinical features, electrocardiogram (EKG) and cardiac magnetic resonance, which showed trace pericardial effusion. The patient's symptom and condition were dramatically improved upon discontinuing mesalamine, and a full recovery was achieved. Mesalamine-induced inflammation of the myocardium (myocarditis) or pericardium (pericarditis) or both (myopericarditis) is rare, but has fatal side effects. Early recognition of these side effects by clinicians and patients is important to prevent progression of the inflammation. Furthermore, patients should be educated to seek urgent medical attention if cardiac symptoms arise.
\end{abstract}

Keywords: Mesalamine; Chest pain; Myopericarditis; Ulcerative colitis; CMR

\section{Introduction}

Inflammation of the myocardium (myocarditis) or pericardium (pericarditis) or both (myopericarditis) as side effects of mesalamine, a drug widely used in the treatment of inflammatory bowel disease (IBD), is a rare, but potentially lethal complication. Early recognition and appropriate intervention are important to prevent progression of the inflammation and avoid adverse cardiovascular outcomes.

Manuscript submitted December 16, 2018, accepted December 27, 2018

aDepartment of Internal Medicine, University of Nevada - Reno, School of Medicine, Reno, NV 89502, USA

${ }^{b}$ Corresponding Author: Mohamed E. Taha, Department of Internal Medicine, University of Nevada - Reno, School of Medicine, Reno, NV 89502, USA. Email: M_tahir1985@yahoo.com

doi: https://doi.org/10.14740/cr820

\section{Case Report}

An 18-year-old Caucasian woman with recently diagnosed ulcerative colitis (UC) presented to our facility with pleuritic chest pain and pre-syncope. The chest pain was sharp with no other accompanying symptoms. She reported no previous infections. She was diagnosed with UC 2 weeks prior to presentation and subsequently started on mesalamine $800 \mathrm{mg}$ four times/day and prednisone $40 \mathrm{mg} /$ day. Past medical history was also significant for anemia related to blood loss.

Physical examination on presentation revealed tachycardia and flow murmur, otherwise unremarkable. Initial 12-lead electrocardiogram (EKG) revealed sinus tachycardia, a heart rate of 108 beats/min and borderline T-wave abnormalities in leads II, III and augmented vector foot (aVF) (Fig. 1).

Laboratory tests revealed an elevated cardiac troponin I of $0.12 \mu \mathrm{g} / \mathrm{L}$ (peak concentration of cardiac troponin I of 5.59 $\mu \mathrm{g} / \mathrm{L}$ ), a C-reactive protein (CRP) concentration of $17.08 \mathrm{mg} /$ $\mathrm{dL}$, (erythrocyte sedimentation rate) ESR of $68 \mathrm{~mm} / \mathrm{h}$, a hemoglobin level of $7.7 \mathrm{~g} / \mathrm{dL}$, and a leukocyte count of $12.3 \mathrm{~K} /$ $\mathrm{uL}$. The rest of lab values were unremarkable on presentation.

Chest X-ray (CXR) revealed hazy bibasilar opacities suggestive of atelectasis, otherwise unremarkable (Fig. 2). An echocardiogram showed normal left ventricular size, thickness, systolic function and diastolic function. The left ventricular ejection fraction was estimated to be $55 \%$ (Fig. 3).

Cardiac magnetic resonance imaging (MRI) with delayed enhancement showed trace pericardial effusion; however, there was no edema in the myocardium, or late gadolinium enhancement in the ventricular wall to suggest scar (Fig. 4). There was no sign of myocardial infarction and thus, the patient was diagnosed with acute myopericarditis.

Given no alternative explanation to patient' symptoms, mesalamine was discontinued. In less than $48 \mathrm{~h}$, her chest pain resolved and serum troponin I trended down dramatically requiring no additional treatment. The patient remained asymptomatic thereafter.

\section{Discussion}

The 5-aminosalicylic acid (5-ASA), also known as mesalamine or mesalazine, and its derivatives remain the mainstay of maintenance therapy for mild forms of IBD, mainly UC $[1,2]$. Sulfasalazine (5-ASA + sulfapyridine) was the original treatment for IBD but was abandoned due to its side-effect 


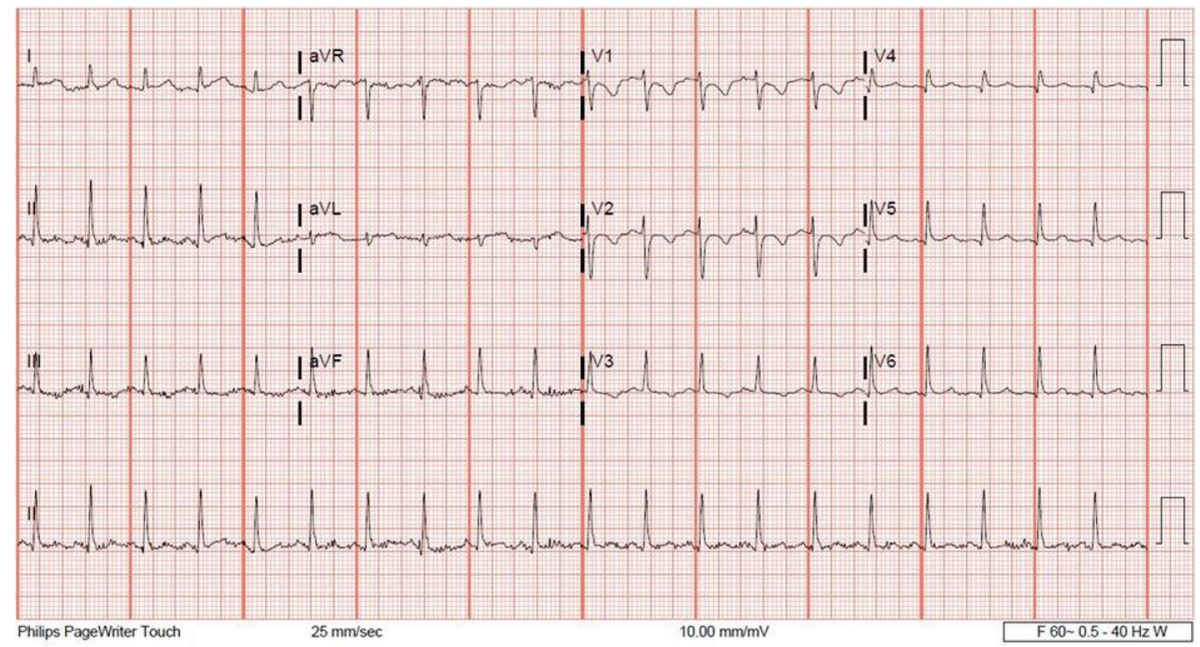

Figure 1. A 12-lead electrocardiogram showing borderline T-wave abnormalities in leads II, III and aVF.

profile caused by sulfapyridine component. Cardiac toxicity in the form of myocarditis, pericarditis, or myopericarditis was rare but lethal adverse effect of sulfasalazine use [3-5]. Mesalamine was introduced in late 1990 as a formulation containing 5-ASA alone with less adverse effects compared to sulfasalazine. Despite the improvement in side-effect profile, cardiac toxicity of 5-ASA continued to be reported in patients receiving mesalamine, which is rare but life threatening [6].

Pathophysiology of 5-ASA related cardiac manifestations is not well understood, and proposed theories include direct toxic effect, humoral or cell mediated mechanisms [7]. The lack of specific pathognomonic features of mesalamine-induced cardiac toxicity and the fact that IBDs themselves can induce similar cardiac manifestations make the diagnosis challenging.

Diagnosis of mesalamine-induced myocarditis and pericarditis is made clinically and involves constellation of cardiac symptoms and laboratory findings. It is mainly based on the temporal relation of those clinical features to initiation of mesalamine therapy. The clinical syndrome is usually witnessed within 1 - 2 weeks since the starting of 5-ASA treatment, and it

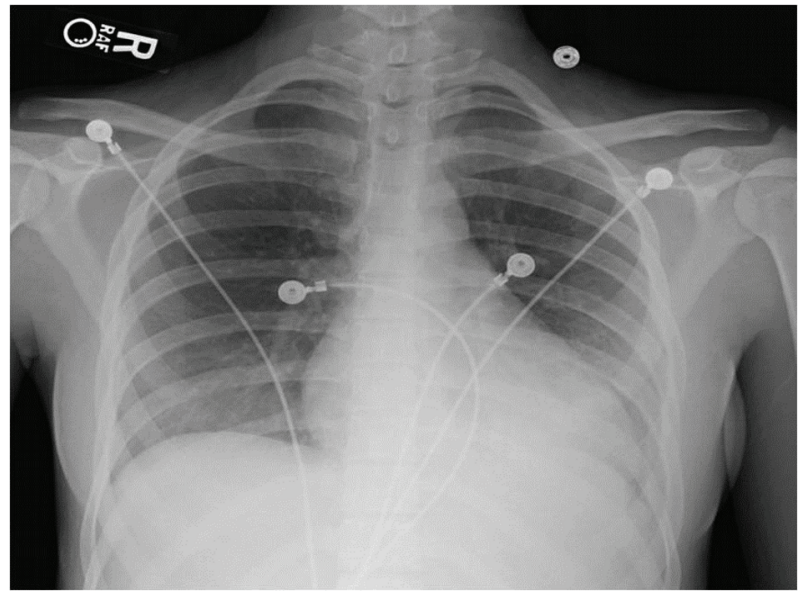

Figure 2. Chest X-ray showing hazy bibasilar opacities suggestive of atelectasis. can present up to 4 weeks or more down the road [8-10]. Classic clinical features include symptoms of fever, chest pain, and dyspnea $[7,11,12]$. Fatigue and weakness are less commonly reported symptoms. Physical exam is usually significant for tachycardia, in addition to pericardial rub in some patients $[7$, 8]. EKG usually reveals nonspecific ST segment changes or T-wave changes, which could be flat, depressed or elevated $\mathrm{T}$ waves, with the latter being more common $[8,9]$.

Laboratory investigations are usually remarkable for elevated white cell count, inflammatory markers (CRP and ESR) and elevated cardiac troponin $[5,8]$. Some cases reported elevated level of N-terminal pro-brain natriuretic peptide [13, 14]. Cardiac imaging informs of echocardiogram or cardiac magnetic resonance can reveal left ventricular systolic dysfunction, in addition to pericardial effusion with or without tamponade effect [7]. Late or delayed contrast enhancement on cardiac MRI was observed in some cases. However, no consistent pattern was noted [15-18].

Several cases of cardiac manifestation related to mesalamine therapy were reported in the literature. Manifestations include myocarditis, pericarditis, plural or pericardial effusion

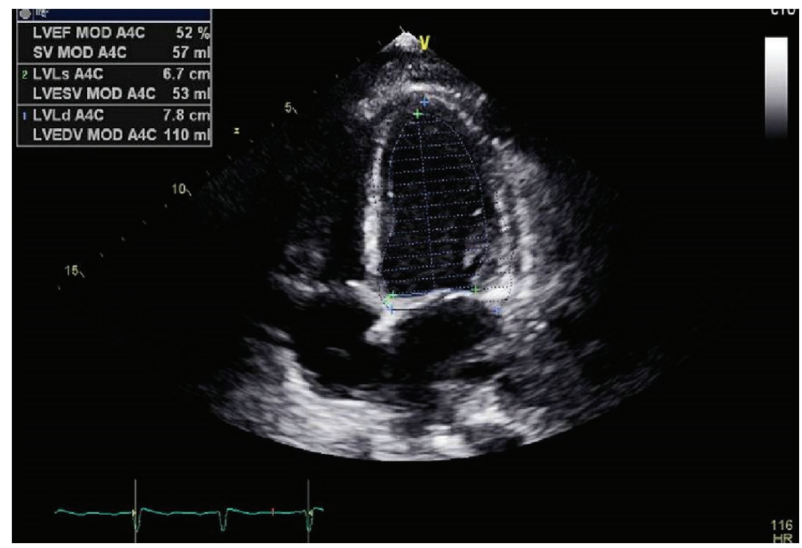

Figure 3. Echocardiogram showing normal left ventricular ejection fraction (LVEF). 


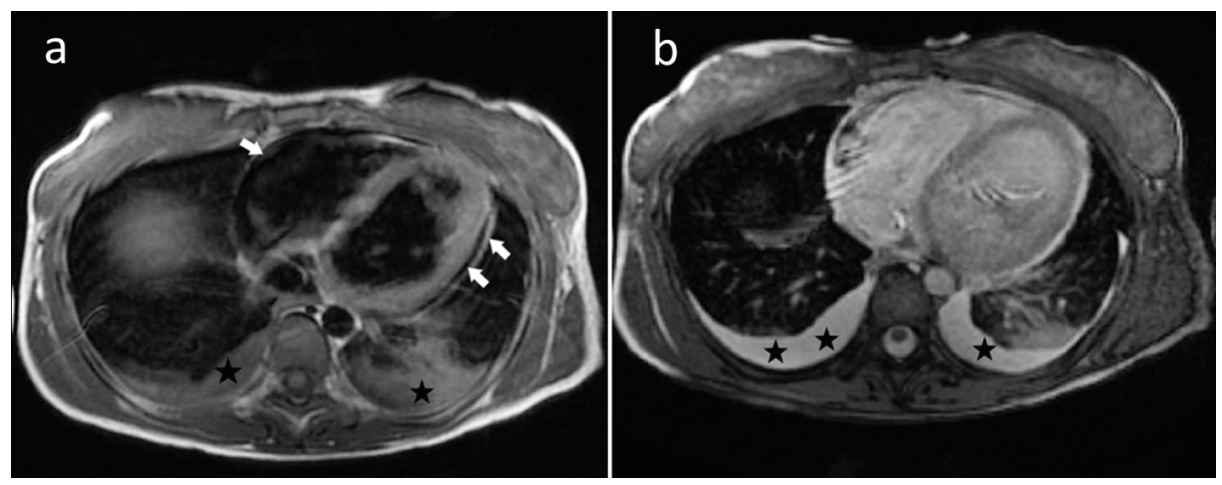

Figure 4. Cardiac magnetic resonance imaging showing trace pericardial effusion (Arrows) and bilateral pleural effusion (Stars).

$[7,11,19,20]$ and recurrent attacks of myopericarditis $[12,21$, 22]. Most of these cases had a favorable outcome after discontinuation of mesalamine therapy that served both as diagnostic and therapeutic interventions. Varying degrees of cardiac workup were pursued in those cases that revealed no evidence of ischemic structural changes. The role of steroid and antiinflammatory therapy remains controversial as most patients improve drastically after discontinuation of mesalamine with rapid resolution of symptoms [6].

Extraintestinal manifestation of IBD can include cardiac complications in form of myocarditis or pericarditis that are seen with both UC and Crohn's disease [23-25]. Such manifestation may present as initial presentations during the course of the disease or may be encountered years after diagnosis. Cardiac toxicity related to IBD remains a rare condition which makes it challenging to diagnose specially in setting of 5-ASA therapy [26]. The temporal relation of the above mentioned clinical features in setting of recent mesalamine therapy initiation should raise suspicion for mesalamine-induced cardiac toxicity.

\section{Conclusions}

Mesalamine-induced inflammation of the myocardium (myocarditis) or pericardium (pericarditis) or both (myopericarditis) is rare, but has fatal side effects. Early recognition of these side effects by clinicians and patients is very important to prevent progression of the inflammation and avoid development of other fatal cardiovascular manifestations. Furthermore, patients should be educated regarding the cardiovascular side effects of mesalamine and advised to seek urgent medical attention if cardiac symptoms arise.

\section{Conflict of Interest}

There is no conflict of interest to declare.

\section{References}

1. Dignass A, Van Assche G, Lindsay JO, Lemann M, Soderholm J, Colombel JF, Danese S, et al. The second Eu- ropean evidence-based Consensus on the diagnosis and management of Crohn's disease: Current management. J Crohns Colitis. 2010;4(1):28-62.

2. Dignass A, Lindsay JO, Sturm A, Windsor A, Colombel JF, Allez M, D'Haens G, et al. Second European evidence-based consensus on the diagnosis and management of ulcerative colitis part 2: current management. J Crohns Colitis. 2012;6(10):991-1030.

3. Clementz GL, Dolin BJ. Sulfasalazine-induced lupus erythematosus. Am J Med. 1988;84(3 Pt 1):535-538.

4. Deboever G, Devogelaere R, Holvoet G. Sulphasalazineinduced lupus-like syndrome with cardiac tamponade in a patient with ulcerative colitis. Am J Gastroenterol. 1989;84(1):85-86.

5. Perrot S, Aslangul E, Szwebel T, Gadhoum H, Romnicianu S, Le Jeunne C. Sulfasalazine-induced pericarditis in a patient with ulcerative colitis without recurrence when switching to mesalazine. Int J Colorectal Dis. 2007;22(9):1119-1121.

6. Brown G. 5-Aminosalicylic Acid-Associated Myocarditis and Pericarditis: A Narrative Review. Can J Hosp Pharm. 2016;69(6):466-472.

7. Kaiser GC, Milov DE, Erhart NA, Bailey DJ. Massive pericardial effusion in a child following the administration of mesalamine. J Pediatr Gastroenterol Nutr. 1997;25(4):435-438.

8. Qanneta R, Garcia Pardo G, Florit Serra L. Acute pleuropericarditis in a patient with primary Sjogren's syndrome and quiescent ulcerative colitis in longstanding 5-aminosalicylic acid therapy. J Crohns Colitis. 2013;7(10):e506507.

9. Vayre F, Vayre-Oundjian L, Monsuez JJ. Pericarditis associated with longstanding mesalazine administration in a patient. Int J Cardiol. 1999;68(2):243-245.

10. Hermida JS, Six I, Jarry G. Drug-induced pericarditis mimicking Brugada syndrome. Europace. 2007;9(1):6668.

11. Jenss H, Becker EW, Weber P. Pericardial effusion during treatment with 5-aminosalicylic acid in a patient with Crohn's disease. Am J Gastroenterol. 1990;85(3):332333.

12. Sentongo TA, Piccoli DA. Recurrent pericarditis due to mesalamine hypersensitivity: a pediatric case report and 
review of the literature. J Pediatr Gastroenterol Nutr. 1998;27(3):344-347.

13. Sposato B, Allegri MP, Riccardi MP, Chigiotti S, Nencioni C, Ricciardi B, Carli T, et al. Mesalazine-induced multi-organ hypersensitivity. Clin Drug Investig. 2010;30(6):413-417.

14. Park EH, Kim BJ, Huh JK, Jeong EH, Lee SH, Bang KB, Seol JS, et al. Recurrent mesalazine-induced myopericarditis in a patient with ulcerative colitis. J Cardiovasc Ultrasound. 2012;20(3):154-156.

15. Baker WL, Saulsberry WJ, Elliott K, Parker MW. Cardiac MRI-confirmed mesalamine-induced myocarditis. BMJ Case Rep. 2015;2015.

16. Galvao Braga C, Martins J, Arantes C, Ramos V, Vieira C, Salgado A, Magalhaes S, et al. Mesalamine-induced myocarditis following diagnosis of Crohn's disease: a case report. Rev Port Cardiol. 2013;32(9):717-720.

17. Garcia-Ferrer L, Estornell J, Palanca V. Myocarditis by mesalazine with cardiac magnetic resonance imaging. Eur Heart J. 2009;30(8):1015.

18. Merceron O, Bailly C, Khalil A, Pontnau F, Hammoudi N, Dorent R, Michel PL. Mesalamine-induced myocarditis. Cardiol Res Pract. 2010;2010.

19. Coman RM, Glover SC, Gjymishka A. Febrile pleuropericarditis, a potentially life-threatening adverse event of balsalazide - case report and literature review of the side effects of 5-aminosalicylates. Expert Rev Clin Immunol. 2014;10(5):667-675.

20. Gujral N, Friedenberg F, Friedenberg J, Gabriel G, Kotler M, Levine G. Pleuropericarditis related to the use of mesalamine. Dig Dis Sci. 1996;41(3):624-626.

21. Sabatini T, Filippini A, Nicosia F, Preti S, Bertoli M. Recurrence of myocarditis after mesalazine treatment for ulcerative colitis: a case report. Inflamm Bowel Dis. 2013;19(3):E46-48.

22. Sonu I, Wong R, Rothenberg ME. 5-ASA induced recurrent myopericarditis and cardiac tamponade in a patient with ulcerative colitis. Dig Dis Sci. 2013;58(8):21482150 .

23. Abu-Hijleh M, Evans S, Aswad B. Pleuropericarditis in a patient with inflammatory bowel disease: a case presentation and review of the literature. Lung. 2010;188(6):505510.

24. Sarrouj BJ, Zampino DJ, Cilursu AM. Pericarditis as the initial manifestation of inflammatory bowel disease. Chest. 1994;106(6):1911-1912.

25. Hyttinen L, Kaipiainen-Seppanen O, Halinen M. Recurrent myopericarditis in association with Crohn's disease. J Intern Med. 2003;253(3):386-388.

26. Cappell MS, Turkieh A. Chronic pericarditis and pericardial tamponade associated with ulcerative colitis. Dig Dis Sci. 2008;53(1):149-154. 